\title{
Effect of Physico-chemical Properties and Weed Management Practices on Weed Dynamics of Dalpatsagar Reservoir, Jagdalpur
}

\author{
Subhash Soni*, A. Pradhan, A. K. Thakur, T. Chandrakar and D. P. Singh \\ S. G. College of Agriculture \& Research Station, IGKV, Jagdalpur (C.G.), India \\ *Corresponding author
}

\begin{abstract}
A B S T R A C T
Dalpatsagar reservoir was severely infested with many aquatic macrophytes throughout the year since last decade and modified the structure of habitat and influenced the aquatic organism. An experiment was conducted in Dalpatsagar reservoir Jagdalpur, Chhattisgarh during February 2019 to February 2020 throughout the year under Randomized Complete

Block Design (RCBD) with three replications comprising seven treatments viz. Glyphosate (41 SL), Paraquat (24 SL) and 2,4-D (amine salt 58\% SL) at 2.00 and 1.00 litre/ha with each herbicides including Absolute control. The herbicides were mixed with sticker (Latron AG-98) and sprayed in three replications by power spray machine (1HP HTP MAK ASPEE) mounted on the boat. The $\mathrm{pH}$ was 6.77 to 6.10 at observation of 3 to 120 days but it gradually increased 7.92 at 300 days of observation. The $\mathrm{pH}$ was lowest 6.40 with Glyphosate @ 2.00 litre/ha, EC was increased $0.59 \mathrm{dSm}^{-1}$ till 300 days and it was significantly higher $\left(0.41 \mathrm{dSm}^{-1}\right)$ value was observed. The value of TDS was also gradually increased $237 \mathrm{mg} / \mathrm{l}$ at 300 days, but after spray of herbicide, the value was reduced $199 \mathrm{mg} / \mathrm{l}$ with application of Glyphosate @ 2.00 litre/ha. $\mathrm{CO}_{3}$ and $\mathrm{HCO}_{3}$ of Dalpatsagar were increased $57.38 \mathrm{mg} / \mathrm{l}$ and $319 \mathrm{mg} / \mathrm{l}$ in response to Glyphosate applied @ 2.00 litre/ha at 300 DAS. The reduction of weed dry weight $\left(\mathrm{g} / \mathrm{m}^{2}\right)$ was maximum with Glyphosate@ 2.00 litre/ha followed by 2,4-D @ 2.00 litre/ha at 20 DAS. Weed control efficiency was significantly higher in Pistia stratiotes $(81.27 \%)$ and Nelumba nucifera $(81.26 \%)$ with glyphosate @ 2.00 litre/ha at 5 and 20 DAS. Mean Weed control efficiency was higher $(63.80 \%)$ with glyphosate @ 2.00 litre/ha with higher cost of control Rs. 6684 per ha followed by 2,4-D @ 2.00 litre/ha. This can be proved that Glyphosate @ 2.00 litre/ha was much effective herbicides to control aquatic weed population followed by 2,4D @ 2.00 litre/ha with Rs. 5747 per ha than other herbicides.
\end{abstract}

\section{Keywords}

Aquatics, Water ecosystem,

Herbicides,

Economics of pond

Article Info

Accepted:

07 September 2020

Available Online:

10 October 2020

\section{Introduction}

Scientifically Aquatic weed is known as aquatic macrophytes those plants are unwanted and important secondary producer for any wetland ecosystem. The wetland are the essential part of biological diversity and ecosystem functioning and its utility performance varied by the hydrological and ecosystem functioning (Banner and MacKenzie, 2000). In natural condition, many of aquatic wildlife such as different fish spices, animal, birds, amphibians, and plant spices (macrophytes and plankton diversity) 
are found in their Natural Habitat. So wetland is a most productive ecosystem they fulfill critical regulatory function of hydrological process within the watershed (Hernandez and Mitsch, 2007; Palit and Mukherjee, 2010). The aquatic weed species is grouped into categories of algae, emergent, submersed and floating species based on their habitat and morphological characteristics (Varshney et al., 2008). Aquatic weed species Alternanthera philoxeroides, Chara spp., Ipomoea spp. Eichhornia crassipes, Hydrilla verticillata, Nelumbo nucifera, Nitella spp. Nymphaea stellata, Salvinia molesta, Typha angustata, Vallisnaria spp. are primary concern in Indian aquatic condition (Gopal and Sharma, 1981). Among these, Eichhornia crassipes, alligator weed and lotus species of aquatic weeds are of primary concern in India and all over world. In general, it is estimated that $20-25 \%$ of the total utilizable water in India is currently infested with Eichhornia crassipes (water hyacinth), while in the state of Assam, Kerala, West Bengal, Orissa and Bihar, more than $40 \%$ water bodies are infested with water hyacinth (Sushilkumar, 2011). Health of any wetland and their ecological functioning are directly related to the survival of any aquatic organisms (Ramesh et al., 2007) and regular monitoring of ecosystem is much needed (Ramachandran et al., 2006). Monitoring of water quality is aimed for the management and conservation of the aquatic habitat by suitable maintaining the physiochemical quality of pond water within the acceptable level (Garg et al., 2010).The Physico-chemical properties such as electric conductivity (EC), $\mathrm{pH}$, Carbonate and Bicarbonate content etc. play a significant role in the composition, distribution, and abundance of aquatic organisms (Mustapha and Omotosho, 2005).

The Dalpatsagar reservoir was constructed by king of Bastar Shree Dalpatdev in 1772 for the purpose of domestic as well as agricultural use for assured water resource by harvesting rain water and store in low lying areas. The lake was constructed with the help of villagers belonging to the kingdom, expanding the area of 3 small ponds into 340.44 acre that sprawled half of the city under the catchment. The important aquatic macrophytes include Ipomoea aquatic, Oryza nivara, Oxalis carniculata, Ipomoea carnea, Typha augustata, Echinodorus grisebachii, Nymphaea alba, Nelumbo nucifera, Nymphaea rubra and Nymphaea indica also found (Pradhan and Patil, 2017). In the integrated weed management practices uses of chemical herbicide is an important tool. In some case use of herbicide is necessary to suppress the aggressive nuisance of vegetation. However, the herbicide is applied as a last option in weed suppression system. In India for controlling various type of submerged and floating weed spices commonly several chemical herbicide like 2,4-D, glyphosate, metsulfuron methyl, paraquat etc. are used in a water bodies (Varshney et al., 2008). Based on environment and system application rate varies and herbicidal efficiency dependent on the specific herbicide and their formulation ((Masser et al., 2013, Whetstone, 2004). In the view of densely infested weed mat on the water surface, manual removal was experienced difficult and costly. The Biological method of control was available only for water hyacinth, but it was considerably more time taking method while other weeds would not be controlled. Therefore, it was necessary to manage the dance weed mat by application of herbicides followed by manual and mechanical removal to reduce the cost of operation by manual method alone. Hence, keeping in the view of above importance, a research programme was conducted to find out the effective weed management practices for infested aquatic weeds population in Dalpatsagar reservoir Jagdalpur 


\section{Materials and Methods}

The research was conducted in aquatic ecosystem during the month of March 2019 to February 2020 at Dalpatsagar reservoir Jagdalpur in Chhattishgarh, India. Dalpat sagar reservoir situated in between $19^{\circ} 5^{\prime} 41^{\prime \prime} \mathrm{N}$ and $82^{\circ} 0$ ' $43^{\prime \prime}$ E with elevation of $563 \mathrm{~m} \mathrm{MSL}$, the water body was severely infested with many type of aquatic weeds. The average annual rainfall of the area is $1400 \mathrm{~mm}$. the major amount of precipitation occurs between June to September (about 3 to 4 months) which is main source of water in aquatic water body. The average annual temperature is during experiment is between $25^{\circ} \mathrm{C}$ to $30^{\circ} \mathrm{C}$ and the hottest and coolest month was May and January respectively were recorded from meteorological observatory S.G. CARS Jagdalpur. The experiment consists of seven treatments with three replications that were laid out in Randomized Complete Block Design (RCBD) by piling bamboo poles at measured distance and one bot (one inch) net was used to confine the aquatic weeds in particular treatments.

The treatments comprised of $\mathrm{T}_{1}$ : Glyphosate 41 SL @ 2.0 litre/ha, T2: Glyphosate 41 SL @ 1.0 litre/ha, $\mathrm{T}_{3}$ : Paraquat $24 \mathrm{SL} @ 2.0 \mathrm{~kg} / \mathrm{ha}$, $\mathrm{T}_{4}$ : Paraquat $24 \mathrm{SL} @ 1.0 \mathrm{~kg} / \mathrm{ha}, \mathrm{T}_{5}: 2,4-\mathrm{D}$ (amine salt 58\%) @ 2.0 litre/ha, T6: 2, 4-D (amine salt 58\%) @ 1.0 litre/ha and $\mathrm{T}_{7}$ : Absolute control (herbicide use restricted). Application of herbicides was done over weed mat dissolving into 500 litre water and added sticker $20 \mathrm{ml}$ (Latron AG-98). The chemical spray was done with power spray machine (1HP HTP MAK ASPEE) mounted on the boat. The experimental area was equally divided into 21 plots of $5 \mathrm{~m}^{2}$ by piling bamboo poles at corners of each plot and separated by net. The gap between replication was 3 meter. Herbicide spraying was done on 31 December 2019.
Water samples of Dalapat sagar reservoir were collected throughout the year at 15 days interval from each plot under different treatments. The samples were analysed for physiochemical properties such as $\mathrm{pH}, \mathrm{EC}$ and TDS (Jackson, 1967) and carbonate \& bicarbonate (Chopra and Kanwar, 1999) using standard procedure. Different types of aquatic weeds were collected and identified during experiment. Treatment wise weed dry weight $\left(\mathrm{g} / \mathrm{m}^{2}\right)$ was recorded after oven drying at $60 \pm 50 \mathrm{C}$ for 72 hours and weed control efficiency was calculated following the standard method. Economics of each treatment was calculated taking into consideration the cost of hiring the boat, machine and labours. Data on density and dry weight of weeds were transformed using square root transformation $(\sqrt{x+0.5})$ before statistical analysis as suggested by Panse and Sukhatme (1967). Observed data studies were subjected to statistical analysis as per the guidelines of Gomez and Gomez (1984). The variance ratio (F-value) was used to test the significance of the treatment effect. Appropriate standard errors and critical difference at $5 \%$ probability level was used to test the statistical significance of the results.

\section{Results and Discussion}

\section{Physio-chemical properties of Dalpatsagr water body \\ pH}

The $\mathrm{pH}$ of Dalpatsagar reservoir was varied with 6.77 to 6.10 at observation of 3 to 120 days and after that the $\mathrm{pH}$ was continuous increases upto 6.23 to 7.92 up to 300 days and ending with increased (Table 1). In weed management treatments, the highest $\mathrm{pH}$ was noted at Absolute control in which herbicide use restricted. The lower value of $\mathrm{pH} 6.64$ was noticed with application of Glyphosate @ 2.00 litre/ha as on weed mat due to fast 
decomposition of weeds, that contributed in biological degradation over the period of time resulting lower $\mathrm{pH}$ with higher dose of Glyphosate.

Although higher $\mathrm{pH}$ was noticed with lower dose of Glyphosate @ 1.00 litre/ha due to summer season during 120 to 150 days showed higher $\mathrm{pH}$ in all the herbicidal treatments whereas lower $\mathrm{pH}$ during winter season because of production of $\mathrm{CO}_{2}$ from biological oxidation process reduced $\mathrm{pH}$ value in the particular treatment (Sharma et al., 1984; Shrinivas and Aruna, 2018).

\section{Electric conductivity (EC)}

The EC of Dalpatsagar reservoir was lowest $\left(0.26 \mathrm{dSm}^{-1}\right)$ at initial 30 Days observation with Absolute control treatment while the highest electric conductivity was recorded $0.59 \mathrm{dSm}^{-1}$ at 240 days under 2,4-D @ 2.00 litre per hectare (Table 2). The value of EC was significantly increased upto 180 days due to dilution of sewage water showing low till 360 days. In weed management treatment EC is higher $\left(0.41 \mathrm{dSm}^{-1}\right)$ with lower dose of 2,4D 1.00 litre per hectare and lowest $\left(0.30 \mathrm{dSm}^{-}\right.$ ${ }^{1}$ ) was recorded for higher dose of Glyphosate @ 2.0 litre/ha.

The maximum electric conductivity (EC) was recorded during summer season and in monsoon season conductivity is gradually decrease is due to increased volume of water as a result of frequent rain that caused a decline in salt concentration (Chandrakiran, 2011).

\section{Total Dissolved Solid (TDS)}

The value of TDS of Dalpatsagar reservoir was significantly differed as advanced in time with season change (Table 3). At the beginning, value of TDS is decreased from $223 \mathrm{mg} / \mathrm{l}$ to $183 \mathrm{mg} / \mathrm{l}$ during 30 to 120 days and later the value of TDS was increased significantly $(237 \mathrm{mg} / \mathrm{l})$ up to 300 days. The significant reduction of TDS was noticed under Glyphosate @ 2.00 litre/ha followed by 2,4-D@2.00 litre per hectare which were $199 \mathrm{mg} / \mathrm{l}$ and $198 \mathrm{mg} / \mathrm{l}$, respectively while lower dose of Paraquat @ $2.00 \mathrm{~kg} / \mathrm{ha}$ and Glyphoste @ 1.00 litre/ha were more effective in reducing TDS over other lower dose of herbicides. Similar study on TDS was quoted by Verma et al., (2012) and Bhatt et al., (1999).

\section{Carbonate $\left(\mathrm{CO}_{3}\right)$ and bicarbonate $\left(\mathrm{HCO}_{3}\right)$ content}

In pond ecosystem higher value of $\mathrm{pH}$ is due to reduced photosynthetic activity and assimilation of $\mathrm{CO}_{2}$ and Bicarbonate during in summer season, which was obvious from the data shown in Table 4 and 5. The availability of Carbonate $\left(\mathrm{CO}_{3}\right)$ was highest $57.38 \mathrm{mg} / \mathrm{l}$ with 2,4-D@ 1.00 litre/ha at 300 days and the lowest $\mathrm{CO}_{3} 43.80 \mathrm{mg} / \mathrm{l}$ under 2,4-D @ 2.00 litre/ha at 120 days. The significantly higher $\mathrm{CO}_{3}$ value $(54.66 \mathrm{mg} / \mathrm{l})$ found with 2,4-D @ 1.00 litre/ha followed by Glyphosate @ 1.0 litre/ ha.

The reduction of $\mathrm{CO}_{3}$ was grater with higher dose of Glyphosate @ 2.00 litre/ha and 2,4-D @ 2.00 litre/ha. The Bicarbonate content was found higher $(319 \mathrm{mg} / \mathrm{l})$ with Glyphosate @ 1.00 litre/ha at 300 days and lowest (246 mg/l) was under 2,4-D @ 2.00 litre/ha at 120 days among all treatments. At lower dose, bicarbonate content was significantly higher (307 mg/l) with 2,4-D @ 1.00 litre/ha. The highest carbonate concentration in September and bicarbonate concentration in April. The higher carbonate and bicarbonate is due to the accumulated organic matter which is produced from decay and decomposition of vegetation and domestic sewage (Shrinivas and Aruna, 2018). 
Table.1 Monthly Variation in $\mathrm{pH}$ of water at Dalpatasgar reservoir

\begin{tabular}{|c|c|c|c|c|c|c|c|c|c|c|c|c|}
\hline \multirow[b]{2}{*}{ Treatment. } & \multicolumn{12}{|c|}{ pH of water at different days of observation } \\
\hline & $\begin{array}{c}30 \\
\text { DAYS }\end{array}$ & $\begin{array}{c}60 \\
\text { DAYS }\end{array}$ & $\begin{array}{c}90 \\
\text { DAYS }\end{array}$ & $\begin{array}{c}120 \\
\text { DAYS }\end{array}$ & $\begin{array}{c}150 \\
\text { DAYS }\end{array}$ & $\begin{array}{c}180 \\
\text { DAYS }\end{array}$ & $\begin{array}{c}210 \\
\text { DAYS }\end{array}$ & $\begin{array}{c}240 \\
\text { DAYS }\end{array}$ & $\begin{array}{c}270 \\
\text { DAYS }\end{array}$ & $\begin{array}{c}300 \\
\text { DAYS }\end{array}$ & $\begin{array}{l}\text { 330* } \\
\text { DAYS }\end{array}$ & $\begin{array}{c}360 \\
\text { DAYS }\end{array}$ \\
\hline T1 & 6.77 & 6.64 & 6.27 & 6.10 & 6.40 & 6.23 & 6.54 & 6.80 & 6.89 & 6.97 & 6.64 & 6.72 \\
\hline $\mathbf{T 2}$ & 7.5 & 7.47 & 7.02 & 6.92 & 7.17 & 7.07 & 7.32 & 7.64 & 7.74 & 7.83 & 7.46 & 7.55 \\
\hline T3 & 7.14 & 7.18 & 6.78 & 6.60 & 6.92 & 6.74 & 7.07 & 7.27 & 7.36 & 7.45 & 7.10 & 7.19 \\
\hline T4 & 7.42 & 7.61 & 6.92 & 6.70 & 7.07 & 6.84 & 7.22 & 7.58 & 7.67 & 7.77 & 7.40 & 7.49 \\
\hline T5 & 6.84 & 6.78 & 6.31 & 6.04 & 6.44 & 6.17 & 6.58 & 6.75 & 6.84 & 6.92 & 6.60 & 6.68 \\
\hline T6 & 7.30 & 7.4 & 6.61 & 6.90 & 6.75 & 7.05 & 6.89 & 7.72 & 7.82 & 7.91 & 7.54 & 7.63 \\
\hline T7 & 7.29 & 7.44 & 6.39 & 6.23 & 6.52 & 6.36 & 6.66 & 6.95 & 7.03 & 7.12 & 6.78 & 6.87 \\
\hline SEm \pm & 0.03 & 0.04 & 0.01 & 0.02 & 0.01 & 0.01 & 0.01 & 0.02 & 0.02 & 0.01 & 0.01 & 0.01 \\
\hline $\mathrm{CD}(\mathrm{P}=0.05)$ & 0.08 & 0.14 & 0.04 & 0.07 & 0.05 & 0.05 & 0.04 & 0.06 & 0.06 & 0.05 & 0.04 & 0.05 \\
\hline
\end{tabular}

* Observation recorded after herbicide application

T1: Glyphosate @ 2.0 litre/ha, T2: Glyphosate @ 1.0 litre/ha, T3: Paraquat @ 2.0 kg/ha, T4: Paraquat @ 1.0 kg/ha, T5:2,4-D @ 2 litre/ha, T6:2,4-D @ 1 litre/ha, $\mathrm{T}_{7}$ : Absolute control

Table.2 Monthly variation in $\mathrm{EC}\left(\mathrm{dSm}^{-1}\right)$ of water at Dalpatasgar reservoir

\begin{tabular}{|c|c|c|c|c|c|c|c|c|c|c|c|c|}
\hline \multirow[b]{2}{*}{ Treatment } & \multicolumn{12}{|c|}{ EC $\left(\mathrm{dS} \mathrm{m}^{-1}\right)$ of water at different days of observation } \\
\hline & $\begin{array}{c}\text { 30 } \\
\text { DAYS }\end{array}$ & $\begin{array}{c}\text { 60 } \\
\text { DAYS }\end{array}$ & $\begin{array}{c}90 \\
\text { DAYS }\end{array}$ & $\begin{array}{c}120 \\
\text { DAYS }\end{array}$ & $\begin{array}{c}150 \\
\text { DAYS }\end{array}$ & $\begin{array}{c}180 \\
\text { DAYS }\end{array}$ & $\begin{array}{c}210 \\
\text { DAYS }\end{array}$ & $\begin{array}{c}240 \\
\text { DAYS }\end{array}$ & $\begin{array}{c}270 \\
\text { DAYS }\end{array}$ & $\begin{array}{c}300 \\
\text { DAYS }\end{array}$ & $\begin{array}{l}\text { 330* } \\
\text { DAYS }\end{array}$ & $\begin{array}{c}360 \\
\text { DAYS }\end{array}$ \\
\hline T1 & 0.27 & 0.30 & 0.31 & 0.40 & 0.31 & 0.48 & 0.45 & 0.45 & 0.44 & 40 & 0.30 & 0.39 \\
\hline T2 & 0.27 & 0.28 & 0.30 & 0.40 & 0.30 & 0.52 & 0.50 & 0.47 & 0.45 & 0.43 & 0.33 & 0.37 \\
\hline T3 & 0.34 & 0.33 & 0.36 & 0.43 & 0.33 & 0.54 & 0.51 & 0.46 & 0.44 & 0.46 & 0.37 & 0.36 \\
\hline T4 & 0.32 & 0.31 & 0.34 & 0.35 & 0.33 & 0.51 & 0.49 & 0.44 & 0.42 & 0.43 & 0.35 & 0.34 \\
\hline T5 & 0.28 & 0.30 & 0.32 & 0.40 & 0.30 & 0.49 & 0.49 & 0.44 & 0.44 & 0.42 & 0.39 & 0.37 \\
\hline T6 & 0.31 & 0.32 & 0.34 & 0.48 & 0.37 & 0.54 & 0.58 & 0.59 & 0.58 & 0.55 & 0.41 & 0.36 \\
\hline T7 & 0.26 & 0.27 & 0.28 & 0.38 & 0.28 & 0.51 & 0.47 & 0.44 & 0.45 & 0.43 & 0.38 & 0.35 \\
\hline SEm \pm & 0.01 & 0.01 & 0.01 & 0.01 & 0.01 & 0.01 & 0.01 & 0.01 & 0.02 & 0.01 & 0.02 & 0.02 \\
\hline $\mathrm{CD}(\mathrm{P}=0.05)$ & 0.03 & 0.02 & 0.02 & 0.03 & 0.03 & 0.03 & 0.02 & 0.03 & NS & NS & NS & NS \\
\hline
\end{tabular}

* Observation recorded after herbicide application

$\mathrm{T}_{1}$ : Glyphosate @ 2.0 litre/ha, $\mathrm{T}_{2}$ : Glyphosate @ 1.0 litre/ha, $\mathrm{T}_{3}$ : Paraquat @ $2.0 \mathrm{~kg} / \mathrm{ha}, \mathrm{T}_{4}$ : Paraquat @ $1.0 \mathrm{~kg} / \mathrm{ha}, \mathrm{T}_{5}: 2,4-\mathrm{D} @ 2$ litre/ha, $\mathrm{T}_{6}: 2,4-\mathrm{D} @ 1$ litre/ha,

$\mathrm{T}_{7}$ : Absolute control 
Table.3 Monthly variation of Total Dissolved Solid (TDS) of water at Dalpatasgar reservoir

\begin{tabular}{|c|c|c|c|c|c|c|c|c|c|c|c|c|}
\hline \multirow[b]{2}{*}{ Treatment } & \multicolumn{12}{|c|}{ TDS (mg/l) at different days of observation } \\
\hline & $\begin{array}{c}30 \\
\text { DAYS }\end{array}$ & $\begin{array}{c}60 \\
\text { DAYS }\end{array}$ & $\begin{array}{c}90 \\
\text { DAYS }\end{array}$ & $\begin{array}{c}120 \\
\text { DAYS }\end{array}$ & $\begin{array}{c}150 \\
\text { DAYS }\end{array}$ & $\begin{array}{c}180 \\
\text { DAYS }\end{array}$ & $\begin{array}{c}210 \\
\text { DAYS }\end{array}$ & $\begin{array}{c}240 \\
\text { DAYS }\end{array}$ & $\begin{array}{c}270 \\
\text { DAYS }\end{array}$ & $\begin{array}{c}300 \\
\text { DAYS }\end{array}$ & $\begin{array}{c}\text { 330* } \\
\text { DAYS }\end{array}$ & $\begin{array}{c}360 \\
\text { DAYS }\end{array}$ \\
\hline T1 & 203 & 199 & 188 & 183 & 192 & 196 & 187 & 204 & 207 & 209 & 199 & 202 \\
\hline $\mathbf{T} 2$ & 214 & 215 & 203 & 198 & 208 & 212 & 202 & 218 & 221 & 224 & 213 & 216 \\
\hline T3 & 225 & 224 & 211 & 208 & 215 & 220 & 212 & 229 & 232 & 235 & 224 & 227 \\
\hline T4 & 223 & 228 & 208 & 201 & 212 & 217 & 205 & 227 & 230 & 233 & 222 & 225 \\
\hline T5 & 205 & 203 & 189 & 181 & 193 & 197 & 185 & 203 & 205 & 208 & 198 & 200 \\
\hline T6 & 219 & 222 & 198 & 207 & 203 & 207 & 211 & 232 & 234 & 237 & 226 & 229 \\
\hline T7 & 219 & 223 & 192 & 185 & 196 & 200 & 191 & 208 & 211 & 214 & 204 & 206 \\
\hline SEm \pm & 2.15 & 1.83 & 1.51 & 0.67 & 1.01 & 1.02 & 1.34 & 1.38 & 1.41 & 1.80 & 1.92 & 1.41 \\
\hline $\mathrm{CD}(\mathrm{P}=\mathbf{0 . 0 5})$ & 5.51 & 5.67 & 4.82 & 2.34 & 3.15 & 3.08 & 4.83 & 4.02 & 4.82 & 5.85 & 5.95 & 4.13 \\
\hline
\end{tabular}

* Observation recorded after herbicide application

$\mathrm{T}_{1}$ : Glyphosate @ 2.0 litre/ha, T 2 : Glyphosate @ 1.0 litre/ha, $\mathrm{T}_{3}$ : Paraquat @ 2.0 kg/ha, T: Paraquat @ 1.0 kg/ha, T5:2,4-D @ 2 litre/ha, T6:2,4-D @ 1 litre/ha,

$\mathrm{T}_{7}$ : Absolute control

Table.4 Monthly variation in Carbonate $\left(\mathrm{CO}_{3}\right)$ content of water at Dalpatasgar reservoir

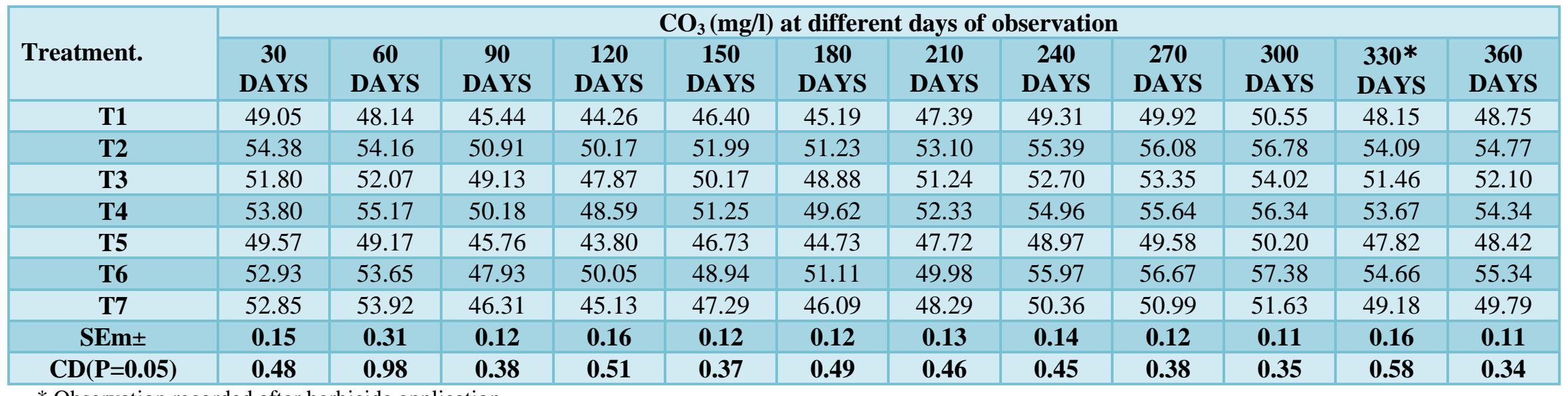

* Observation recorded after herbicide application

$\mathrm{T}_{1}$ : Glyphosate @ 2.0 litre/ha, $\mathrm{T}_{2}$ : Glyphosate @ 1.0 litre/ha, $\mathrm{T}_{3}$ : Paraquat @ 2.0 kg/ha, T : Paraquat @ 1.0 kg/ha, T $: 2,4-\mathrm{D} @ 2$ litre/ha, T $: 2,4-\mathrm{D}$ @ 1 litre/ha,

$\mathrm{T}_{7}$ : Absolute control 
Table.5 Monthly variation in Bicarbonate $\left(\mathrm{HCO}_{3}\right)$ content of water at Dalpatasgar reservoir

\begin{tabular}{|c|c|c|c|c|c|c|c|c|c|c|c|c|}
\hline \multirow[b]{2}{*}{ Treatment. } & \multicolumn{12}{|c|}{$\mathrm{HCO}_{3}(\mathrm{mg} / \mathrm{l})$ at different days of observation } \\
\hline & $\begin{array}{c}30 \\
\text { DAYS }\end{array}$ & $\begin{array}{c}60 \\
\text { DAYS }\end{array}$ & $\begin{array}{c}90 \\
\text { DAYS }\end{array}$ & $\begin{array}{c}120 \\
\text { DAYS }\end{array}$ & $\begin{array}{c}150 \\
\text { DAYS }\end{array}$ & $\begin{array}{c}180 \\
\text { DAYS }\end{array}$ & $\begin{array}{c}210 \\
\text { DAYS }\end{array}$ & $\begin{array}{c}240 \\
\text { DAYS }\end{array}$ & $\begin{array}{c}270 \\
\text { DAYS }\end{array}$ & $\begin{array}{c}300 \\
\text { DAYS }\end{array}$ & $\begin{array}{c}\text { 330* } \\
\text { DAYS }\end{array}$ & $\begin{array}{c}360 \\
\text { DAYS }\end{array}$ \\
\hline T1 & 276 & 271 & 255 & 249 & 261 & 254 & 266 & 277 & 281 & 284 & 271 & 274 \\
\hline $\mathbf{T 2}$ & 306 & 304 & 286 & 282 & 292 & 288 & 298 & 311 & 315 & 319 & 304 & 308 \\
\hline T3 & 291 & 293 & 276 & 269 & 282 & 275 & 288 & 296 & 300 & 304 & 289 & 293 \\
\hline T4 & 302 & 310 & 282 & 273 & 288 & 279 & 294 & 309 & 313 & 317 & 302 & 305 \\
\hline T5 & 279 & 276 & 257 & 246 & 263 & 251 & 268 & 275 & 279 & 282 & 269 & 272 \\
\hline T6 & 297 & 302 & 269 & 281 & 275 & 287 & 281 & 315 & 319 & 323 & 307 & 311 \\
\hline T7 & 297 & 303 & 260 & 254 & 266 & 259 & 271 & 283 & 287 & 290 & 276 & 280 \\
\hline SEm \pm & 1.11 & 2.02 & 1.93 & 1.24 & 1.31 & 1.42 & 1.03 & 1.69 & 1.73 & 3.01 & 1.32 & 1.67 \\
\hline $\mathrm{CD}(\mathrm{P}=0.05)$ & 3.48 & 6.18 & 4.42 & 3.81 & 4.72 & 4.22 & 4.08 & 5.19 & 5.68 & 10.08 & 4.31 & 5.93 \\
\hline
\end{tabular}

* Observation recorded after herbicide application

$\mathrm{T}_{1}$ : Glyphosate @ 2.0 litre/ha, $\mathrm{T}_{2}$ : Glyphosate @ 1.0 litre/ha, T $:$ Paraquat @ 2.0 kg/ha, T 4 : Paraquat @ 1.0 kg/ha, T5:2,4-D @ 2 litre/ha, T $: 2,4-\mathrm{D} @ 1$ litre/ha,

$\mathrm{T}_{7}$ : Absolute control

Table.6 Effect of weed control treatments on weed control efficiency and economics

\begin{tabular}{|c|c|c|c|c|c|c|c|c|c|c|c|c|c|c|c|c|}
\hline \multirow{3}{*}{$\begin{array}{c}\text { Treatme } \\
\text { nt }\end{array}$} & \multicolumn{15}{|c|}{ Weed Control Efficiency (\%) } & \multirow{3}{*}{$\begin{array}{c}\text { Cost of } \\
\text { weed } \\
\text { control } \\
(\text { Rs. })\end{array}$} \\
\hline & \multicolumn{2}{|c|}{$\begin{array}{c}\text { Eichhornia } \\
\text { crassipes }\end{array}$} & \multicolumn{2}{|c|}{ Pistia stratiotes } & \multicolumn{2}{|c|}{$\begin{array}{l}\text { Ipomoea } \\
\text { aquatica }\end{array}$} & \multicolumn{2}{|c|}{$\begin{array}{c}\text { Nelumbo } \\
\text { nucifera }\end{array}$} & \multicolumn{2}{|c|}{$\begin{array}{c}\text { Alternanthera } \\
\text { philoxeroides }\end{array}$} & \multicolumn{2}{|c|}{$\begin{array}{c}\text { Nymphaea } \\
\text { rubra }\end{array}$} & \multicolumn{2}{|c|}{$\begin{array}{c}\text { Cyperus } \\
\text { javanicus }\end{array}$} & \multirow[t]{2}{*}{ Mean } & \\
\hline & $\begin{array}{c}5 \\
\text { DAS }\end{array}$ & $\begin{array}{c}20 \\
\text { DAS }\end{array}$ & $\begin{array}{c}5 \\
\text { DAS }\end{array}$ & $\begin{array}{c}20 \\
\text { DAS }\end{array}$ & $\begin{array}{c}5 \\
\text { DAS }\end{array}$ & $\begin{array}{c}20 \\
\text { DAS }\end{array}$ & $\begin{array}{c}5 \\
\text { DAS }\end{array}$ & $\begin{array}{c}20 \\
\text { DAS }\end{array}$ & $\begin{array}{c}5 \\
\text { DAS }\end{array}$ & $\begin{array}{c}20 \\
\text { DAS }\end{array}$ & $\begin{array}{c}5 \\
\text { DAS }\end{array}$ & $\begin{array}{c}20 \\
\text { DAS }\end{array}$ & 5 DAS & $\begin{array}{c}20 \\
\text { DAS }\end{array}$ & & \\
\hline $\mathbf{T 1}$ & 30.03 & 78.01 & 65.27 & 81.27 & 51.25 & 70.31 & 33.57 & 81.26 & 29.89 & 58.15 & 52.81 & 75.53 & 76.59 & 76.30 & 63.80 & 6684 \\
\hline $\mathbf{T 2}$ & 17.20 & 55.14 & 52.15 & 69.06 & 37.53 & 57.01 & 26.00 & 64.58 & 13.60 & 45.38 & 28.23 & 54.56 & 50.49 & 45.37 & 45.45 & 5626 \\
\hline T3 & 27.05 & 65.50 & 51.22 & 74.63 & 40.72 & 61.57 & 28.13 & 65.44 & 17.71 & 47.94 & 31.36 & 53.92 & 56.19 & 52.78 & 50.51 & 5697 \\
\hline T4 & 5.78 & 38.88 & 27.06 & 64.54 & 12.47 & 35.34 & 3.23 & 45.66 & 6.52 & 3.93 & 8.39 & 31.25 & 21.79 & 29.63 & 23.89 & 5347 \\
\hline T5 & 28.69 & 76.78 & 52.52 & 80.27 & 41.69 & 62.48 & 34.52 & 69.79 & 23.94 & 51.67 & 50.91 & 66.31 & 72.89 & 74.35 & 51.70 & 5747 \\
\hline T6 & 12.28 & 61.91 & 43.55 & 68.79 & 36.01 & 50.09 & 21.67 & 63.13 & 11.76 & 33.40 & 20.79 & 53.07 & 39.31 & 13.43 & 37.80 & 5280 \\
\hline T7 & 0.00 & 0.00 & 0.00 & 0.00 & 0.00 & 0.00 & 0.00 & 0.00 & 0.00 & 0.00 & 0.00 & 0.00 & 0.00 & 0.00 & 0.00 & 0.00 \\
\hline SEm \pm & 0.75 & 0.38 & 3.54 & 1.67 & 2.65 & 2.20 & 0.37 & 3.98 & 3.06 & 2.57 & 0.61 & 5.44 & 2.87 & 10.95 & - & 235 \\
\hline $\begin{array}{c}\text { CD } \\
(P=0.05)\end{array}$ & 3.01 & 2.51 & 14.05 & 6.64 & 10.53 & 7.84 & 2.44 & 13.83 & 7.18 & 8.22 & 1.93 & 9.32 & 6.81 & 43.52 & - & 987 \\
\hline
\end{tabular}

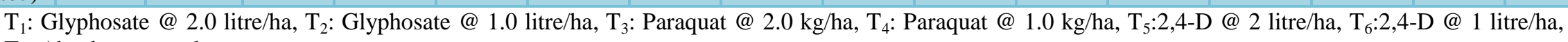

$\mathrm{T}_{7}$ : Absolute control 


\section{Weed Control Efficiency and economy}

Weed control efficiency was significantly higher in Pistia stratiotes $(81.27 \%)$ and Nelumba nucifera (81.26\%) with Glyphosate @ 2.00 litre/ha whereas the lowest weed control efficiency was observed (Table 6) in Alternanthera philoxeroides $(3.93 \%)$ under Paraquat at lower dose $(1 \mathrm{~kg} / \mathrm{ha})$ on 20 DAS. At 5 and 20 DAS, Glyphosate @ 2.00 litre/ha gave higher $(63.80 \%)$ weed control efficiency followed by 2,4-D @ 2.00 litre/ha with response to all the weed species. The lowest weed control efficiency was observed with lower dose of Paraquat $(1.00 \mathrm{~kg} / \mathrm{ha})$. The weed control efficiency was increased with Eichhornia crassipes (30.03 and 78.01), Pistia stratioides (65.27 and 81.27) and Ipomoea aquatica (51.21 and $81.26 \%$ ) at 5 and 20 DAS, respectively with Glyphosate @ 2.00 litre/ha. The higher cost of weed control (Rs. 6684 per ha) was recorded significantly with Glyphosate @ 2.00 litre/ha followed by 2,4-D @ 2.00 litre/ha (Rs. 5747 per ha). Lower dose of Glyphosate (1.00 litre/ha) and 2,4-D (1.00 litre/ha) were (Rs. 5626 per ha and Rs. 5280 per ha respectively) found approximately similar in cost of weed control. Whereas, lowest cost of control was observed with lower dose of 2,4-D (1.00 litre/ha).

\section{References}

Banner, A. and MacKenzie, W. 2000. The ecology of wetland ecosystem. 45 extension note. Ministry of Forest Research Program, Victoria, BC.

Bhatt, I.R., Lacoul, P., Lekhale, H.D. and Jha, P.K. 1999. Physico- chemical characteristics and phytoplanktons of Taudaha lake Kathmandu. Poll.Res., 18(4): 353- 358.

Chandrakiran. 2011. Impact of sediment characteristics on the benthic communities of lake Mansar. Ph. D Thesis, University of Jammu, Jammu.
Chopra S. L. and Kanwar J. S. 1999. Analytical Agricultural Chemistry, Kalyani Publishers, New Delhi. 4th Ed. PP:280-283.

Chinnusamy, C., Janaki, P., Murali Arthanari, P. and Muthukrishnan, P. 2012. Effect of Post-emergence Herbicides on Water hyacinth (Eichhornia crassipes)Tank Culture Experiments Pak. J. Weed Sci. Res., 18: 105-111

Datta.S. 2009. Aquatic Weeds and Their Management for Fisheries https://www.researchgate.net/publicati on/258931408 p22.

Garg, R.K., Rao, R.J., Uchchariya, D., Shukla, G., and Saksena, D.N. 2010. Seasonal variations in water quality and major threats to Ramsagar reservoir, India. Afr. J. Environ. Sci. Tech., 4(2): 061-076.

Gomez, K.A. and Gomez, A.A. 1984. Statistical procedures for Agricultural Research. A Willey.

Gopal, B. and Sharma, K.P. 1981. Water Hyacinth (Eichhornia Crassipes) the Most Troublesome Weeds of the World. Hindasia Publisher, New Delhi, 129 p.

Hernandez, M.E. and Mitsch, W.J. 2007. Denitrifcation in created riverine wetlands: influence of hydrology and season. Ecol Eng 30:78-88.

Jackson, M.L. (1973). Soil Chemical Analysis, Prenctice Hall of India Pvt. Ltd., New Delhi, pp. 183-204.

Masser, M.P., Murphy, T.R. and Shelton, J.L. 2013. Aquatic weed management: herbicides. Southern regional aquaculture center. (http://srac.tamu.edu/index.cfm/event/g etFactSheet/whichfactsheet/66/.html)

Mustapha, M.K. and Osotosho, J.S., 2005. An Assessment of the physico-chemical Properties of Moro Lake, Kwara state Nigeria, African Journal of Applied Zoology \& Environment Biology, Vol.7 P.73-77. 
Palit, D., and Mukherjee, A. 2010. Characterization of Physico-chemical properties of water and soil in Lalb and hafresh water wetland in Birbhum District West Bengal. Ecology Environment and Conservation, 15(4) 239-245.

Panse, V.G. and Sukhatme, P.V. 1967. Statistical methods of agricultural workers. Indian Council of Agricultural Research, New Delhi

Pradhan, A. and Patil, S.K. 2017. Conference: Wetland Wealth of Chhattisgarh Dalpatsagar, Chhattisgarh State Planning Commission Raipur.

Ramachandra, T.V., Rishiram, R., and Karthick, B. 2006. Zooplankton as Bioindicators: Hydro-Biological investigations in selected Bangalore Lakes. Technical Report Centre for Ecological Sciences, Indian Institute of Science. Bangalore. Pp: 115.

Ramesh, M., Saravanan, M., and Pradeepa, G. 2007. Studies on the physicochemical characteristics of the Singallunar Lake, Coimbatore, India. In: Proceeding of National Seminar on Limnology. Maharana Pratap University of Agriculture and Technology, Udaipur, India.
Sharma, M.S., Sharma, L.L. and Durve, V.S., 1984. Eutrophication of Lake Pichhola in Udaipur, Rajasthan. Poll. Res., 3(2): 39-44.

Srinivas, M. and Aruna, M. 2018. Physicochemical analysis of a lake, Erea Cheruvu in Siddipet District of Telangana State, India. International Journal of Recent Scientific Research Vol. 9, Issue, 10(E), pp. 29420-29425.

Sushilkumar. 2011. Biological based chemical integration for early control of water hyacinth. Indian Journal of Weed Science 43 (3\&4): 211-214.

Varshney Jay G, Sushilkumar and Mishra J.S. 2008. Current status of aquatic weeds and their management in India. Proceedings of Tal: (Eds. Sengupta M. and Dalwani R.) The 12 World Lake Conference, Jiapur: 1039- 1045.

Verma P.U., Purohit A.R. and Patel N.J. 2012. Pollution Status of Chandlodia Lake Located In Ahmedabad Gujarat, IJERA, 2, 1600-1606.

Whetstone, J. M. 2004. Aquatic weed control overview. Clemson Cooperative Extension. (http://www.clemson.edu/extension/hgi c/plants/pdf/hgic1714.pdf)

\section{How to cite this article:}

Subhash Soni, A. Pradhan, A. K. Thakur, T. Chandrakar and Singh, D. P. 2020. Effect of Physico-chemical Properties and Weed Management Practices on Weed Dynamics of Dalpatsagar Reservoir, Jagdalpur. Int.J.Curr.Microbiol.App.Sci. 9(10): 747-755. doi: https://doi.org/10.20546/ijcmas.2020.910.090 